The chain of arguments for the new approach is both stepwise and convoluted. We are taken through descriptions of quasi-species, error thresholds, multidimensional sequence space, and hypercycles in the reconstruction of the path that led to self-sustaining cell-like organisms. Each step requires precise integration into the earlier ones, and only through continual reiteration of concepts can the entire formulation avoid collapse. Eigen accomplishes this by removing the burden of explanation of many molecular biological concepts and terminology from the main body of the text and places them in both a series of separate "Vignettes" and extended sections of footnotes and glossary. Readers can therefore digest the advocacy at a variety of levels: the experienced molecular biologist will be able to read it straight through, whereas non-biologists can jump back and forth between the central argument and supplementary descriptions. Either way, the discussion is lucid, allowing a crystallization of understanding.

Steps Towards Life, which is an English translation of a work first published in 1987 in German, is unequivocally a promotion of a viewpoint that stands out in a field of study nearly as variegated as the prebiotic milieu. The quasi-species is the primary innovation, being a population of molecules of similar composition. The frequency distribution of this population is determined by each molecule's relative replication efficiency. As such, the quasispecies as a unit is subject to selective forces, in contrast to standard populationgenetics models in which a wild type is predominant and all variants respond independently to selection.

It is clear how the two alternatives would fare in accommodating the origins of life. However, it is less apparent that the quasi-species concept is best - and perhaps only - applicable to acellular evolution. Eigen makes extensive use of viruses as contemporary examples of quasi-species, and moreover augments his presentation with concrete experiments done in his laboratory that are in vitro accounts of quasi-species evolution. Consequently, an unobservant reader may miss the brief disclaimer restricting quasispecies theory to single-gene evolution. The epigenetic interactions that play such an important role in shaping the evolution of complex organisms are not discussed, an oversight that would be of no importance if the contrast between darwinian and quasi-species evolution during the origin of life were not so strongly made. Yet in the book we are warned that darwinians are likely to defend the patterns we have come to expect in other evolutionary contexts. This is undoubtedly true.

In condensing more than 20 years of theory and experiment into a short and engaging volume, Eigen makes the quasispecies and related concepts accessible to all scientists and thereby allows broad peer review and general consideration of his revolutionary ideas.

Niles Lehman is in the Department of Molecular Biology, The Scripps Research Institute, 10666 North Torrey Pines Road, La Jolla, California 92037, USA

\section{Flower power}

\section{G. R. Moore}

Pigment of the Imagination: A History of Phytochrome Research. By Linda C. Sage. Academic: 1992. Pp. 562. $£ 66, \$ 99.50$.

Phytochrome is a blue-green pigment that acts as a plant photoreceptor protein in the mediation of many light-activated processes including seed germination, leaf formation, circadian rhythms and flowering. Such physiological processes are often sensitive to the quality, quantity or duration of light. For example, flowering of many plants is controlled by the length of day: some plants (such as lettuce and spinach) flower better under conditions of long days and short nights, whereas others (such as chrysanthemums) flower only as the days shorten (the critical factor is, in fact, the period of darkness). Horticulturists often exploit these responses, for example, by exposing chrysanthemums to a brief flash of light during the night to delay flowering until Christmas.

But precisely how the absorption of light by phytochrome results in these various activities is still not fully understood, although it has been established that the protein is photoreversible, existing in two interchangeable forms with respect to absorption, a red and a far-red form; a blue chromophore, phytochromobilin, is responsible for its activation when exposed to red light, and this photoconversion is reversible on absorption of far-red light. It is also known that there are several different species of phytochrome and that the protein determines some responses through the regulation of gene expression.

Linda Sage provides a thoroughly researched historiographical account of phytochrome research during the past 80 years, from the initial discovery that plants are able to detect daylength, through the extraction of the lightsensitive pigment, to the biophysical, biochemical and molecular genetic work of more recent years. The text is profusely illustrated with figures, tables and photographs of buildings, equipment and key researchers. The author writes in a clear style and makes good use of footnotes to explain the science where necessary and to present important details of experiments. The result is a book that can be followed easily by nonspecialists.

In adopting a historiographical approach, Sage has had the difficult task of blending scientific facts and data with accounts of the personalities and careers of the researchers involved. The result can sometimes prove to be frustrating and compromised. For example, I was eager to learn the structure of phytochromobilin and how it attaches to its apo-protein. Although this is discussed at several points in the book, the sections are not cross-referenced well in the text, making it more difficult to grasp the current state of knowledge than if all the relevant information had been brought together in one section (there is a clear index, however). Also disappointing is the account of Garner and Allard's work. These two researchers collaborated for more than 20 years and provided the first clear demonstration of photoperiodism in plants. Yet despite the joint nature of their work it was Garner who was given much of the credit by journalists and fellow scientists for this discovery, a situation which, according to Sage, naturally displeased Allard. But exactly who did what is not made clear, and we do not get to learn Garner's thoughts on the subject. The most we are treated to is the statement that "Garner and Allard maintained outwardly cordial relations despite this tension". This sketchy treatment is a shame, because Sage has generally done a considerable amount of research, analysing scientific literature and personal records, and interviewing many people.

The publishers describe the phytochrome story as "a paradigm for the process of scientific discovery". Indeed, what Sage does very well is to remind us that scientific research builds on the work of people in a variety of disciplines, and not just those in one field. Phytochrome research provides splendid examples of the advantages of such collaboration: an understanding of the mechanisms of phytochrome action required the development of molecular biology techniques to allow the regulation of gene transcription to be studied; and an accurate characterization of plants using action spectra required the development of light filters. Too many researchers today take this collaborative nature of scientific research for granted. For this reason, the book deserves a wider audience than just photobiologists, plant physiologists and historians of science.

G. R. Moore is in the School of Chemical Sciences, University of East Anglia, Norwich NR4 7TJ, UK.

NATURE · VOL 359 - 15 OCTOBER 1992 